\title{
On the subject of drug addiction. The use of profiling for the examination of drug abuser's behaviour
}

\author{
In tema di tossicodipendenza. \\ La tecnica del profiling, per la disamina del comportamento del tossicodipendente \\ En relación con la toxicomanía. La técnica del profiling para la valoración \\ del comportamiento del toxicómano
}

\author{
Silvia Badiali, ${ }^{1}$ Monica Calderaro ${ }^{2}$ \\ ${ }^{1}$ Dottoressa Magistrale in Investigazione, Criminalità e Sicurezza Internazionale, Università degli Studi Internazionali \\ di Roma (UNINT); ${ }^{2}$ Grafologa forense, Criminologa, Dottore in Psicologia ad indirizzo clinico, Responsabile Didattico \\ e Docente del Corso di Formazione in Grafologia Forense, Sapienza Università di Roma, Italy
}

\begin{abstract}
By the use of profiling and the analysis of the deviant behavioural conduct in general terms, the figure and the behaviour of the drug abuser is examined in his peculiar features as well. In this way a behavioural model reference could rise in those cases in which there is an addicted person or a presumed one. In this paper are also introduced the New Psychoactive Substances (NPS), new drugs that cause important psychological and psychical consequences on individuals who are used to consume them. Therefore, thanks to this focus on familiar, social, environmental and psychopathological causes of the drug abuser, we try to understand his possible relation with criminality and if some initial signals could be identified in order to allow us to recognize an addicted person, introducing in this regard another useful device, the psychographic examination. Finally, also new kind of addictions are here presented together with how they could widen this study, including the particular epidemic situation caused by Covid-19.
\end{abstract}

\section{RIASSUNTO}

Tramite la tecnica del profiling e l'analisi della condotta comportamentale deviante in termini generali, viene successivamente esaminata la figura e il comportamento del tossicodipendente e le sue caratteristiche peculiari, tali da far emergere un modello comportamentale di riferimento nei casi in cui ci si trovi di fronte ad una persona dipendente o presunta tale. In questo elaborato vengono anche introdotte le Nuove Sostanze Psicoattive (NPS), nuove droghe che causano delle importanti conseguenze psichiche e psicologiche nell'individuo che ne fa uso. Inoltre grazie ad un focus sulle cause famigliari, sociali, ambientali e psicopatologiche del tossicodipendente si cerca di comprendere il suo possibile legame con la criminalità e se sia possibile individuare alcuni segnali iniziali che permettano di riconoscere una persona dipendente, introducendo così un altro utile strumento, ossia la disamina psicografologica. Infine vengono prese in esame le nuove forme di dipendenza e come queste influiscano in questa panoramica, considerando nell'analisi anche la particolare condizione pandemica causata dal Covid-19.

\section{RESUMEN}

A través de la técnica del profiling y del análisis conductual general se analizan la figura y el comportamiento del toxicómano y también sus peculiaridades para hacer surgir un modelo de comportamiento de referencia útil cuando se encuentre una persona con dependencia real o supuesta. En esta composición se presentan también las Nuevas Sustancias Psicoactivas (NPS), nuevas drogas que causan importantes consecuencias psíquicas y psicológicas en el individuo que las utiliza. Además, gracias a la observación de causas familiares, sociales, ambientales y psicopatológicas del toxicómano se intenta de comprender su potencial relación con la criminalidad y si existe la posibilidad de identificar algunas señales iniciales que permiten el reconocimiento de la persona dependiente, introduciendo otro instrumento útil: la valoración psicográfica. Por último, se analizan las nuevas tipologías de dependencia y como estas influyen en este contexto, tomando en consideración también la particular situación pandémica provocada por el Covid-19.

\section{Introduzione}

Il profiling si occupa principalmente di esaminare una determinata condotta comportamentale, in particolare della persona deviante, ponendo l'accento su quegli elementi che spingono il soggetto verso il compimento di atti criminali. La maggior parte degli individui viene educata secondo aspettative generali comuni, e sebbene talvolta possano presentarsi dei comportamenti scorret- ti, questi non sono necessariamente pericolosi per la comunità o i singoli individui. Tuttavia, il comportamento di alcune di queste persone devia significativamente dalla norma e questo può comportare serie problematiche, poiché la loro condotta è riconosciuta come una violazione delle norme sociali principali (Palermo G., Mastronardi V., 2005, pag. 40). Oltre a concentrarsi sulla condotta comportamentale deviante in termini generali, questo lavoro di analisi si concentra in modo più approfondito sulla figura del tossicodipendente e le sue caratteristiche peculiari, tali da far emer- 
gere un modello di riferimento utile a spiegare cosa spinga questi individui a percorrere la strada della dipendenza, sia da sostanze stupefacenti, sia in riferimento alle dipendenze comportamentali. Comprendere le cause che conducono alla dipendenza quindi è senz'altro un elemento indispensabile e per tale motivo sono state considerate le cause famigliari, sociali, ambientali e psicopatologiche. Inoltre nell'eventualità di poter elaborare un possibile profilo comportamentale del tossicodipendente, vengono analizzati anche i segnali della dipendenza, i quali permettono di riconoscere eventuali "spie" in grado di classificare il soggetto come dipendente o presunto tale. All'interno del contesto della tossicodipendenza vengono citate le Nuove Sostanze Psicoattive (NPS) che stanno ormai condizionando alcune categorie di soggetti nella loro quotidianità, a causa dei gravi danni psichici e psicologici che sono in grado di generare e per le quali si sta rilevando un aumento progressivo all'interno dei mercati illegali. Un ulteriore strumento utile al possibile profiling del tossicodipendente è la disamina psicografologica, in grado di poter evidenziare una condotta dipendente o presunta tale. Inoltre è doveroso ricordare che oggi, quando si parla di dipendenze, non si fa più riferimento solo alle sostanze stupefacenti, bensì anche ad altre forme di dipendenza, alcune delle quali si sono accentuate proprio durante l'attuale situazione pandemica da Covid-19.

\section{Cos'è il profiling}

Il termine "profiling" indica l'insieme di quei presidi operativi atti ad accertare con strumenti e discipline adeguati, tipologie, autore e vittima di reato. Il "profilo" quindi è il risultato del lavoro di profiling ed è un elemento vantaggioso ed indispensabile per quei crimini all'apparenza inesplicabili ed a prima vista immotivati (Palermo G., Mastronardi V., 2005, pag. 46).

L'utilizzo del profiling si diffuse con il caso del dinamitardo "Mad Bomber", un elettricista e meccanico americano che per sedici anni, negli anni '40 e '50, terrorizzò New York con esplosivi che fabbricò per colpire luoghi perlopiù pubblici (Palermo G., Mastronardi V., 2005, pag. 47). Non riuscendo a fermare la serie di attentati che stava scuotendo gli Stati Uniti, la polizia di quegli anni si rivolse allo psichiatra James A. Brussel che, esaminando le fotografie scattate sui luoghi degli attentati e l'intero caso, trasse delle conclusioni significative e delineò il profilo psicologico relativo al tipo di criminale che si nascondeva dietro questa serie di atti criminali. Chiamato ad illustrare la tecnica da lui utilizzata, lo psichiatra spiegò che, attraverso il metodo induttivo, ossia l'osservazione di alcuni caratteri specifici, giunse ad una conclusione più ampia, cioè ad una descrizione generale delle caratteristiche del soggetto in grado di compiere gli attentati in questione (Douglas J., 2017, pag. 30-31).

Non tutti i crimini però richiedono necessariamente un'attività di profiling. Perciò per giustificare un profiling, sarebbe necessario trovarsi di fronte a tre elementi principali, così come indicato in Palermo G., Mastronardi V., 2005:

1. serialità di atti criminali (stupro, omicidio, molestie sessuali);

2. atti criminali con mancata identificazione del colpevole;

3. possibile recidivismo.

L'attività di profiling si concentra nel dare risposte precise a specifiche domande: "che cosa" fa riferimento all'analisi della scena del delitto; "perché" alla motivazione del colpevole; "chi" è il soggetto stesso che ha commesso il reato; "quando" è il momento in cui è avvenuto il fatto, ma soprattutto perché è stato scelto quel preciso momento dal colpevole e se il delitto potrebbe ripetersi in futuro (Palermo G., Mastronardi V., 2005, pag. 50).
Perché al profiler serve esaminare una determinata condotta comportamentale?

Lo scopo principale dell'attività di profiling è la cattura del colpevole, ma oltre a questo anche il cercare di spiegare il comportamento criminale del soggetto e la motivazione profonda di questo impulso. Capendo il suo comportamento è possibile poi tracciare i punti principali della sua personalità e, nel migliore dei casi, a prevenire che determinati soggetti (principalmente seriali) commettano ancora crimini violenti. L'attività di profiling è diventata ormai indispensabile a causa dei cambiamenti avvenuti nel panorama criminale; in passato la maggior parte dei crimini avveniva fra persone legate tra loro e i moventi scaturivano da sentimenti esasperati come collera, gelosia, vendetta, perciò per risolvere $i$ delitti era necessario risolvere il problema emotivo che li aveva scatenati. Al contrario negli ultimi anni, sono diventati sempre più numerosi i reati commessi da e verso sconosciuti, e ciò comporta che in molti casi non è presente un reale movente su cui poter lavorare. Infatti a motivare questi soggetti sono meccanismi ben più complessi e tale complessità rende più intricato il modello comportamentale che si può affiancare a sentimenti comuni a quelli suindicati (Douglas J., 2017, pag. 26).

Sulla base di questa analisi sul profiling, è stato preso in esame il tossicodipendente attraverso un focus sulla sua personalità ed il suo comportamento, al fine di delineare i principali aspetti che lo caratterizzano e cercando di comprendere le motivazioni che spingono tale soggetto a scegliere la tossicodipendenza come stile di vita.

\section{In tema di tossicodipendenza}

La "tossicodipendenza" viene definita dall'Organizzazione Mondiale della Sanità (OMS) come una malattia ad andamento cronico-recidivante che spinge l'individuo, in maniera più o meno coatta, ad assumere sostanze (droghe) a dosi crescenti o costanti per avere temporanei effetti benefici soggettivi, la cui persistenza è indissolubilmente legata alla continua assunzione della sostanza. Oggi questo termine viene utilizzato con minor frequenza, dal momento in cui il contesto si è mano a mano arricchito di molte altre dipendenze, alcune delle quali non prevedono sostanze tossiche. Per questo motivo si preferisce utilizzare il termine "dipendenza patologica"l che avendo carattere generale, riesce ad includere l'intero contesto delle dipendenze.

In Italia, negli ultimi anni, risulta sempre più frequente anche l'utilizzo del termine "addiction", dal latino addictus che significa schiavo, e che in lingua inglese viene impiegato per indicare proprio una determinata dipendenza, ossia una condotta attraverso la quale una persona viene resa appunto schiava. L'espressione infatti, è entrata nel linguaggio comune italiano da quando è stata introdotta l'ultima versione del Manuale Diagnostico e Statistico dei disturbi mentali (DSM, aggiornato alla versione 5 dell'anno 2013), che ha mantenuto il termine inglese, non trovando una soluzione soddisfacente per la traduzione del corrispettivo termine italiano. ${ }^{2}$

Solitamente la dipendenza da droghe è un disturbo cronico recidivante che si caratterizza di tre elementi principali: la compulsione

\footnotetext{
1 Per "dipendenza patologica" si intende una forma morbosa determinata dall'uso distorto di una sostanza, di un oggetto o di un comportamento. Una condizione invasiva correlata ad un'abitudine incontrollabile e irrefrenabile che il soggetto non riesce ad allontanare da sé (Caretti V., La Barbera D., 2005, pag. 11).

2 https://magazine.istitutoeuropeodipendenze.it (vedi Sitografia).
} 
nel ricercare ed assumere la sostanza; la perdita del controllo quando si cerca di limitarne l'assunzione; la presenza di uno stato emotivo negativo (disforia, irritabilità, ansia) quando non si ha più l'accesso libero alla sostanza (Di Giannantonio M., Girardi P., 2016, pag. 32).

È necessario puntualizzare che il consumo di sostanze stupefacenti nel corso degli ultimi anni è un fenomeno sempre più crescente e sta piano piano assumendo tratti preoccupanti, uno dei quali è l'introduzione delle droghe all'interno della normale vita quotidiana, soprattutto dei giovani. Tali comportamenti dipendenti sono dovuti ad una ricerca quasi compulsiva del piacere immediato, al fine di spezzare la catena di regolarità e noia che comporta la vita di tutti i giorni. Difatti, il consumo ha carattere perlopiù ricreativo ed occasionale ed è legato a ritualità notturne come la discoteca, le quali sono vissute all'insegna della trasgressività e dello "sballo" (Caretti V., La Barbera D., 2005, pag. 236-237). Molte di queste sostanze utilizzate dai giovani possono causare gravi danni psicologici e psichici, anche perché recentemente sono state introdotte sul mercato le Nuove Sostanze Psicoattive (NPS). Esse sono sostanze di origine sintetica caratterizzate da una struttura chimica di base, la quale viene continuamente modificata in laboratorio da parte di esperti appartenenti ad organizzazioni criminali, al fine di eludere i controlli delle forze dell'ordine e per evitare che tali sostanze possano essere introdotte all'interno delle tabelle delle sostanze illegali. Tali sostanze sono progettate per creare i medesimi effetti delle droghe cosiddette "classiche", come cocaina, eroina, cannabis, LSD, MDMA e negli ultimi anni la quantità di questo tipo di sostanze è cresciuta significativamente. ${ }^{3}$ Il termine "nuove" non significa necessariamente che ci si trovi di fronte a sostanze completamente originali, bensì a sostanze che solo recentemente sono diventate disponibili sul mercato. ${ }^{4}$ La maggior parte delle NPS viene sintetizzata fuori dall'Europa, specialmente in Cina e India ed esse vengono poi vendute in punti vendita al dettaglio o tramite Internet, il quale risulta oggi il canale di vendita prediletto, poiché grazie ai vari forum presenti su siti web appositi, è possibile scambiare informazioni sull'ampia varietà di sostanze disponibili (Di Giannantonio M., Girardi P., 2016, pag. 150). A mano a mano che le NPS vengono identificate vengono inserite all'interno delle Tabelle Nazionali delle Sostanze Stupefacenti e Psicotrope, aggiornate tramite Decreto ministeriale e approvate dal Ministero dell'Interno. Nelle quattro Tabelle sono elencate le sostanze stupefacenti poste sotto il controllo nazionale e internazionale. La prima Tabella comprende: oppio e derivati oppiacei come morfina, eroina, metadone; foglie di Coca e derivati; amfetamina e derivati amfetaminici come ecstasy; allucinogeni come LSD, mescalina, ketamina, fenciclidina. La seconda Tabella fa riferimento alla cannabis. La terza Tabella ai barbiturici, farmaci che agiscono sul sistema nervoso centrale e hanno effetti sedativi e anestetici. La quarta Tabella fa riferimento alle benzodiazepine, classe di psicofarmaci ansiolitici con effetti ipnotici, anestetici. Inoltre è presente anche la Tabella dei medicinali, nella quale sono inseriti quei medicinali a base di sostanze attive stupefacenti di impiego terapeutico e ad uso umano o veterinario, e il loro relativo regime di dispensazione. ${ }^{5}$ Il decreto del 6 agosto 2021 ha aggiornato la prima e la quarta Tabella. Esso ha come oggetto l'inserimento di nuove sostanze psicoattive in considerazione ai casi di intossicazione acuta registrati sul territorio nazionale nel periodo aprile-settembre 2020 e i sequestri operati dalle forze dell'ordine in Italia nel periodo luglio-ottobre 2020 e tenuto conto del parere favorevole del Ministro della Salute per ridurre i rischi connessi all'assunzione e diffusione di queste sostanze sul territorio nazionale. Pertanto nel decreto vi è l'elenco di sostanze come la metallilescalina, l'isopropilfenidato, le quali sono riconducibili per struttura a molecole presenti nella prima Tabella, e sostanze come clonazolam, bromazolam, le quali invece sono riconducibili per struttura a molecole pre- senti nella quarta Tabella e possono essere considerate di fatto delle nuove sostanze psicoattive. ${ }^{6}$ Recentemente vi è stato un ulteriore aggiornamento delle Tabelle I e IV sopracitate con il decreto del 2 dicembre 2021, tenuto conto delle segnalazioni di nuove molecole pervenute nel primo semestre dell'anno 2021 da parte dell'Unità di coordinamento del Sistema nazionale di Allerta precoce del Dipartimento Politiche Antidroga della Presidenza del Consiglio dei Ministri che arricchiscono l'elenco delle nuove sostanze psicoattive. ${ }^{7}$

Tra le NPS individuate e consumate con maggior frequenza ed elencate all'interno del sito del Ministero dell'Interno vi troviamo:

- I Cannabinoidi Sintetici (CS), i quali hanno come principio attivo la pianta della cannabis e vengono chiamati generalmente Spice o Dream. Solitamente vengono venduti come incensi o profumatori ambientali, dichiarando sulla confezione un contenuto diverso rispetto a quello effettivamente presente Sono molto pericolosi, poiché i consumatori li utilizzano pensando che siano delle sostanze naturali alternative alla cannabis, mentre in realtà producono effetti ben più dannosi rispetto a quest'ultima (Di Giannantonio M., Girardi P., 2016, pag. 151); - I Catinoni sintetici, derivano dal catinone, ossia una molecola naturale presente nella pianta del Quat (pianta originaria delle regioni orientali dell'Africa) che ha azione psicostimolante. Essi sono venduti sotto forma di compresse di vari generi e colori, in capsule o in cristalli, facendoli passare per "sali da bagno" o "fertilizzanti per piante". ${ }^{8}$ Il catinone sintetico più noto è il mefedrone, generalmente ingerito o sniffato e promosso come droga ricreazionale $;^{9}$

- Le fenetilamine (4-MEC e MDPV) sono una classe di molecole ad azione psicoattiva. Anch'esse sono vendute sotto forma di compresse, polvere e cristalli, anche se in Italia numerosi sequestri sono stati sotto forma di francobolli, detti blotters; ${ }^{10}$

- La ketamina è una molecola di origine sintetica che possiede proprietà anestetiche e analgesiche e talvolta può essere utilizzata come "rape drug", poiché induce disinibizione e uno stato onirico. Nel mercato illecito essa viene venduta come liquido o sotto forma di polvere cristallina. ${ }^{11}$ All'interno di questa categoria vi è anche la fenciclidina (PCP), chiamata polvere d'angelo e consumata oggi per gli effetti dissociativi che provoca nell'individuo (Di Giannantonio M., Girardi P., 2016, pag. 156);

- Le piperazine rappresentano una classe molto ampia di composti chimici e sono molecole che hanno visto un potenziale farmaceutico, ma che infine non hanno mai raggiunto effettivamente il mercato. Esse sono note come sostanze stimolanti e la più nota tra queste è la benzilpiperazina $(\mathrm{BZP} ;)^{12}$

- Il Fentanil, un potente analgesico narcotico il quale comporta una forte dipendenza in chi ne fa uso;

- Gli oppioidi sintetici, tra i quali il metorfano, sono sostanze simili alla morfina e causano nella persona che ne abusa gratificazione e piacere; ${ }^{13}$
https://onlinelibrary.wiley.com/ (vedi Sitografia).
https://antidroga.interno.gov.it (vedi Sitografia).
https://www.salute.gov.it (vedi Sitografia).
https://www1.ordinemediciroma.it (vedi Sitografia).
https://www1.ordinemediciroma.it (vedi Sitografia).
http://allerta.dronetplus.eu (vedi Sitografia).
https://www.interno.gov.it (vedi Sitografia).
Ibidem.
http://allerta.dronetplus.eu (vedi Sitografia).
2 http://allerta.dronetplus.eu (vedi Sitografia).

13 Ibidem. 
- Da non sottovalutare in questo campo, anche i prodotti medicinali da prescrizione come: l'ossicodone, oppioide con effetti sedativi; le benzodiazepine; gli antipsicotici come l'olanzapina; gli antidpressivi come il bupropione (Di Giannantonio M., Girardi P., 2016, pag. 149);

- L'acido gammaidrossibutirico (GHB), noto in gergo come "scoop" o "ecstasy liquida" può essere utilizzato come droga da stupro, poiché può venir aggiunto alle bevande senza che se ne possa rilevare la presenza, essendo insapore e inodore e permette ai criminali di soggiogare la volontà dei consumatori, inducendo stati di semi incoscienza e amnesia, se non addirittura coma; ${ }^{14}$

- Recentemente è stato notato un incremento del consumo di sostanze note come "Performance and Image Enhancing Drugs" (PIED). Queste sostanze sono composti che includono anche supplementi alimentari come vitamine o ormoni o estratti vegetali, e sono utilizzate per aumentare la massa muscolare, o la funzione sessuale o migliorare l'intelligenza (Di Giannantonio M., Girardi P., 2016, pag. 149).

La lista sempre più crescente di questo nuovo tipo di sostanze ha portato i vari Stati ad aggiornare frequentemente i vari database, anche se purtroppo molte NPS sono in grado di eludere i controlli internazionali, dal momento in cui molte di queste sostanze vengono vendute su Internet a prezzi contenuti. In un articolo del Forensic Research \& Criminology International Journal, gli esperti Connie Smith e Ohagan Andrew, riferiscono infatti che queste nuove sostanze psicoattive sono perlopiù utilizzate da coloro che presentano già da tempo una dipendenza da sostanze. ${ }^{15}$ Difatti attualmente la figura del tossicomane è quella del poliabusatore e tra le motivazioni principali di tale condizione, si possono trovare: l'automedicazione, ossia quando il soggetto prova da solo a trovare dei rimedi che possano in qualche modo alleviare sintomi come stress, ansia, tuttavia rendendosi conto solo successivamente di essere rimasto intrappolato nel ciclo dell'abuso; il potenziamento degli effetti delle singole sostanze, come nel caso delle NPS; l'uso meramente ricreativo; il tentativo di contrastare, attraverso il consumo di una nuova sostanza, gli effetti indesiderati causati dalla sostanza assunta in precedenza. ${ }^{16}$

Per ciò che concerne il comportamento del tossicodipendente, vi sono delle fasi e cicli che lo accompagnano nella sua esperienza con la sostanza e da cui si possono comprendere molti dei comportamenti adottati. La sua esperienza con la sostanza può essere suddivisa in tre stadi: il primo è chiamato dell'incontro o della "luna di miele", ed in tale stadio non emergono ancora quei segnali tipici che indichino la presenza di una forte dipendenza con la sostanza, ma vi è solamente un senso di benessere generalizzato; il secondo stadio è quello intermedio, chiamato anche delle dosi crescenti, poiché l'individuo, mantenendo costante il dosaggio della sostanza, sente svanire piano piano gli effetti iniziali e per poter ricreare tali medesimi effetti aumenta la dose di sostanza assunta, difatti si può assistere ad un tipo di comportamento "depravato" da parte del soggetto, il quale è orientato alla ricerca compulsiva della sostanza; infine il terzo stadio è quello delle ripetute disintossicazioni o della "porta girevole", nel quale il soggetto, a seguito di una presa di coscienza circa la sua condizione, decide di rivolgersi a centri per la disintossicazione. Tuttavia durante questa fase si può assistere a varie ricadute nel consumo di droga e quindi un ritorno alla cura e così via (Di Giannantonio M., Girardi P., 2016, pag. 104). Pertanto nell'analisi del comportamento del tossicodipendente è necessario tenere a mente questi stadi, poiché spiegano a grandi linee da cosa sia causato il suo atteggiamento ed aiutano ad avere un quadro più dettagliato e complessivo della condotta di tale persona dipendente; ciò può essere senz'altro d'aiuto nella delineazione di un possibile profilo comportamentale di quest'ultima.

\section{Tossicodipendenza e criminalità}

In questo capitolo viene messo a fuoco il possibile legame tra la tossicodipendenza ed eventuali atteggiamenti criminali dovuti ad essa. Cosa spinge i soggetti tossicodipendenti a commettere reati?

Si possono distinguere quattro categorie di reati correlati all'abuso di sostanze stupefacenti: ${ }^{17}$

- i reati psicofarmacologici sono quelli scaturiti da un consumo cronico di sostanze psicoattive e ciò può innescare nell'individuo episodi di violenza e aggressività. I meccanismi secondo $i$ quali queste sostanze attivino i comportamenti devianti non sono ancora ben determinati e compresi, tuttavia molte droghe sintetiche utilizzate hanno effetti stimolanti, producendo effetti psicotici che spesso possono intensificare eventuali problemi comportamentali pregressi;

- i reati economici compulsivi fanno riferimento al costo della dipendenza, che spinge alcuni individui a commettere atti criminali (rapine, taccheggi, furti) al fine di ottenere il denaro necessario per acquistare la droga;

- $\quad$ i reati sistemici che implicano atti violenti (omicidio o violenza all'integrità fisica della persona), spesso commessi da individui che appartengono a determinate organizzazioni criminali che si occupano della distribuzione della droga;

- i reati contro la legge in materia di stupefacenti, con i quali si fa riferimento al consumo, alla detenzione, alla coltivazione, alla produzione, al traffico di sostanze e dove il nesso tra i due elementi precedentemente citati non è causato dal soggetto stesso, bensì dal legislatore.

Per poter comprendere appieno l'eventuale rapporto tra comportamenti devianti e tossicodipendenza e il comportamento stesso del tossicodipendente è però altrettanto interessante individuare $\mathrm{i}$ possibili segnali della dipendenza.

Il primo fattore rilevante da considerare quindi è la compulsività, detta craving. Essa deriva da uno stato di forte disagio del soggetto nel quale si fa strada un senso di vuoto sempre più pressante che dà origine a stati d'animo caratterizzati da rabbia, ostilità, solitudine. Tale condizione può essere scatenata da stimoli particolari e del tutto inaspettati come situazioni, luoghi, persone (Di Giannantonio M., Girardi P., 2016, pag. 16). Un altro ulteriore segnale è l'astinenza, che fa riferimento ad un'interruzione o riduzione significativa della quantità di sostanza assunta abitualmente. Solitamente infatti, l'assunzione di droga fa sì che l'organismo si adatti alla presenza della sostanza, perciò una volta che tale assunzione viene interrotta bruscamente, si può creare uno squilibrio interno che viene avvertito dal soggetto e che causa sintomi sgradevoli. ${ }^{18}$ Infine vi è la tolleranza, un meccanismo che porta il soggetto ad aumentare la dose della sostanza stupefacente, al fine di ottenere lo stesso effetto degli inizi, cercando di mantenere la stessa intensità di risultato. Tra i vari meccanismi che consentono di incrementare la dose, vi è quello di cambiare la via d'assunzione: passare per esempio dalla via orale alla via endovenosa. ${ }^{19}$

Ulteriori cofattori interessanti ed indispensabili da considerare quando si guarda alla figura del tossicodipendente nell'eventualità di stilare un profilo comportamentale di riferimento, sono le cause famigliari, ambientali, sociali e psicopatologiche.

\footnotetext{
4 Ibidem.

15 https://medcraveonline.com (vedi Sitografia).

6 https://www.cattolicanews.it (vedi Sitografia).

17 https://www.emcdda.europa.eu (vedi Sitografia).

$18 \mathrm{https}: / /$ magazine.istitutoeuropeodipendenze.it (vedi Sitografia).

19 Ibidem.
} 
Le relazioni sociali, specialmente per gli adolescenti, possono essere un fattore estremamente complesso, poiché da una parte rappresentano un possibile salvagente per l'individuo che si trova in una situazione di difficoltà, ma dall'altra possono essere la causa stessa delle numerose problematicità che derivano dall'abuso di sostanze. L'ambiente si collega automaticamente al fattore sopradescritto, poiché in alcuni contesti devianti, nei quali l'unica risposta è ed è sempre stata la sostanza, la ricerca di altri stimoli appare quasi irrilevante per coloro che sono abituati ad un'unica realtà. In alcuni Paesi infatti il consumo di sostanze è molto elevato e vi è una tolleranza culturale alla tossicodipendenza. ${ }^{20}$ La famiglia poi rappresenta il primo nucleo nel quale il soggetto è cresciuto, perciò il suo interfacciarsi con l'ambiente circostante e le relazioni sociali che ha intrapreso, sono il frutto delle dinamiche famigliari a cui egli ha assistito. Pertanto quando si mira alla delineazione di un profilo di una persona tossicodipendente, basilare è l'analisi della famiglia dalla quale esso proviene. Invero uno dei fenomeni altrettanto diffuso è la tossicodipendenza causata da abusi e maltrattamenti nell'ambito famigliare: in questo caso la sostanza viene percepita come una cura a tutto il dolore subito in famiglia, il più delle volte causato da persone care. Spesso infatti i casi di abuso e maltrattamento possono innescare sensi di colpa nella persona che li ha subiti, portandola ad adottare atteggiamenti autodistruttivi o devianti che le permettono di sfogare la frustrazione e la rabbia, avallando l'idea che la sostanza possa in qualche modo "curare" il danno subito. "Che cosa cerca di guarire il tossicomane quando cerca e fa uso di sostanze?" (Caretti V., La Barbera D., 2005, pag. 323). Una possibile risposta si pensa che stia nel fatto che la droga cerchi di ristabilire una regolazione con un contatto oramai danneggiato, dovuto ad una basalità dell'esistenza stessa che non è una condizione scatenante la tossicodipendenza, bensì una condizione appartenente allo stare nel mondo del soggetto (Caretti V., La Barbera D., 2005, pag. 324). Infine quando si parla di cause psicopatologiche si fa riferimento a tutti quei fattori collegati ai disturbi mentali e alle patologie ad essi connessi. Correlato a tali cause vi è il concetto di "doppia diagnosi", termine che indica la comorbilità tra disturbi da uso di sostanze stupefacenti e disturbi psichiatrici, quest'ultimi classificabili mediante il DSM-5. Inoltre da un punto di vista analitico, esso ci permette di differenziare il tossicodipendente psichiatrico grave, da un tossicodipendente generico, ossia colui che non presenta alcun disturbo mentale. In campo psichiatrico si può effettivamente parlare di casi di doppia diagnosi quando è presente più di un disturbo specifico in un determinato soggetto durante un periodo di tempo ben definito (Di Giannantonio M., Girardi P., 2016, pag. 23). All'interno delle cause psicopatologiche si può comprendere anche la dimensione di "novelty seeking": essa consiste nella ricerca continua della novità, tipica dei soggetti portati a sperimentare un forte eccitamento alla presenza di stimoli nuovi ed inaspettati. Nei contesti adolescenziali i comportamenti orientati verso la suddetta dimensione sono molto frequenti e, mentre da un lato questa è del tutto normale con la fase tipica adolescenziale, dall'altro un significativo numero di individui sono sempre più indirizzati verso l'attuazione di esperienze rischiose. Ad ogni modo, la copresenza di un disturbo di personalità si associa all'abuso di sostanze anche in base ad alcune caratteristiche particolari dell'individuo come l'età di inizio della condotta tossicomane e il livello di funzionamento globale dell'organismo dell'individuo. ${ }^{21}$

In merito alle conseguenze psicopatologiche dovute al consumo di droghe classiche e NPS da parte di un soggetto, così come riportato da Di Giannantonio M., Girardi P., 2016, unitamente agli articoli di Rinaldi R. et al., 2020, sulle NPS e di Canoniero S., Maremmani I., Pacini M., 2002, ed infine sulla base di quanto ricavato dal DSM-5, esse sono state riassunte nella Tab. 1.
Alla luce delle considerazioni sopraesposte in merito alla tossicodipendenza, è senz'altro necessario per chi opera all'interno di questo settore, approfondire le strategie che si possono attuare, al fine di preventivare un comportamento dipendente da parte di un individuo. Lo strumento più efficace è certamente la prevenzione, la quale però deve essere attuata conoscendo il contesto in cui si sviluppa maggiormente il fenomeno della tossicodipendenza.

Per quanto riguarda il contesto italiano, nel "Piano Nazionale della Prevenzione 2020-2025" viene indicata la prevenzione come strumento utile ed efficace per contrastare il fenomeno della tossicodipendenza. È necessario prediligere approcci multicomponenti che possano favorire il coordinamento di diversi settori e ridurre la domanda, modificando gli atteggiamenti. Dato il problema così consistente, la prevenzione non può essere affidata esclusivamente al sistema sociosanitario, bensì è altrettanto fondamentale coinvolgere le diverse istituzioni presenti, al fine di garantire un intervento complessivo e sinergico. Oltre a puntare sulle abilità individuali del soggetto quindi, è necessario intervenire anche sul suo ambiente di vita (come la famiglia e la comunità). ${ }^{22}$

Sul piano Europeo, il Consiglio dell'Unione Europea il 18 Dicembre 2020 ha approvato un documento che ha per oggetto la "Strategia dell'UE in materia di droga per il periodo 2021-2025". L'attuazione delle misure presenti in tale scritto dovrebbe garantire un elevato livello di protezione, sicurezza e stabilità e contribuire a sensibilizzare sulla tematica delle tossicodipendenze. Innanzitutto basilare è ridurre l'offerta di droga attraverso il rafforzamento della sicurezza: ciò comprende la prevenzione e la dissuasione delle attività criminali connesse alla droga da parte della criminalità organizzata, attraverso una cooperazione tra le varie autorità giudiziarie. Dopodiché il documento si concentra sul contrasto al contrabbando di droga all'interno e all'esterno dell'UE, ponendo l'attenzione sulle frontiere che non fanno parte dei canali consolidati. In aggiunta, come già evidenziato analizzando le NPS, è necessario monitorare i mercati digitalizzati nei quali le sostanze illecite sono più facilmente acquistabili, anche per i prezzi meno elevati. Inoltre sebbene alcuni Stati membri utilizzino delle misure coercitive per gli individui in possesso di sostanze illegali o che fanno uso di quest'ultime, alcuni di questi non considerano reato il consumo o il possesso di droga. In questo settore quindi, sono necessari strumenti più completi e approfonditi per lo scambio di informazioni ed esperienze tra $i$ vari Stati. ${ }^{23}$

A livello internazionale un importante organo di controllo delle sostanze stupefacenti è l'International Narcotics Control Board (INCB), che ha il compito di monitorare l'attuazione dei trattati internazionali sulle droghe. Esso è operativo dal 1968 ed è tecnicamente indipendente dai governi e dalle Nazioni Unite, infatti i suoi 13 membri rivestono l'incarico a titolo personale e sono eletti dall'Economic and Social Council e possono richiedere pareri agli esperti dell'OMS. L'organo è incaricato di valutare i requisiti di legittimità delle sostanze controllate e impedire che finiscano all'interno del mercato illecito. Oggi sta assumendo un ruolo sempre più ampio, poiché riferisce circa i trend del narcotraffico e commenta le politiche intraprese dagli stati membri dell'ONU. ${ }^{24}$

\footnotetext{
20 https://www.iss.it (vedi Sitografia).

21 https://www.iss.it (vedi Sitografia).

22 https://www.salute.gov.it (vedi Sitografia).

23 https://www.politicheantidroga.gov.it (vedi Sitografia).

24 https://idpc.net (vedi Sitografia).
} 
Tab. 1. Conseguenze psicopatologiche derivanti dal consumo di droghe classiche e nuove sostanze psicoattive.

Droghe classiche (cocaina, eroina, morfina, LSD, MDMA...) Nuove sostanze psicoattive (NPS)

Disturbo antisociale di personalità

(Canoniero S., Maremmani I., Pacini M., 2002;

American Psychiatric Association, DSM-5, 2014)

Disturbo borderline di personalità

(Canoniero S., Maremmani I., Pacini M., 2002;

American Paychiatric Asoociation, DSM-5, 2014)

Alterazione della percezione, principalmente fenomeni illusionali

e allucinazioni

(Di Giannantonio M., Girardi P., 2016)

Amnesia dissociativa

(Di Giannantonio M., Girardi P., 2016; American Psychiatric

Association DSM-5, 2014)

Disturbo bipolare di tipo I e II

(Di Giannantonio M., Girardi P., 2016; American Psychiatric

Association, DSM-5, 2014)

Frequenza di componenti depressive

(Canoniero S., Maremmani I., Pacini M., 2002)

Disturbo d'ansia generalizzata e fobica, in particolare agorafobia

e attacchi di panico veri e propri

(Di Giannantonio M., Girardi P., 2016)

Comportamenti dissociativi con alterazioni della coscienza dell'Io, all'interno del DSM-5, riferito ai disturbi dissociativi

(Di Giannantonio M., Girardi P., 2016; American Psychiatric

Association, DSM-5, 2014)

Comportamenti stereotipati o ripetitivi causati dall'esperienza

paranoide

(Di Giannantonio M., Girardi P., 2016)

Disturbo percettivo persistente da allucinogeni (flashback)

(Di Giannantonio M., Girardi P., 2016)
Disturbi psicotici come allucinazioni uditive e visive, ideazione paranoidea

(Di Giannantonio M., Girardi P., 2016; Rinaldi R. et al., 2020;

American Psychiatric Association, DSM-5, 2014)

Ideazione suicidaria, che si può trovare all'interno del disturbo depressivo maggiore

(Di Giannantonio M., Girardi P., 2016;

American Psychiatric Association, DSM-5, 2014)

Disturbo d'ansia generalizzata

(Di Giannantonio M., Girardi P., 2016; American Psychiatric

Association, DSM-5, 2014)

Sindrome serotoninergica possibile

(Di Giannantonio M., Girardi P., 2016)

Dissociazione, all'interno dei disturbi dissociativi

(Di Giannantonio M., Girardi P., 2016; American Psychiatric

Association, DSM-5, 2014)
Alterazione del tono dell'umore con fenomeni di aggressività, irritabilità, rabbia

(Di Giannantonio M., Girardi P., 2016)

In alcuni casi aumento delle prestazioni sessuali

(Di Giannantonio M., Girardi P., 2016)

Sindrome delirante, all'interno del disturbo delirante

(Rinaldi R. et al., 2020; American Psychiatric Association, DSM-5, 2014)

Anestesia dissociativa

(Rinaldi R. et al., 2020)

\section{Il profiling del tossicodipendente}

In questo capitolo viene introdotto un importante strumento utile nell'evidenziazione di un comportamento di tipo dipendente: la disamina psicografologica. La grafologia è una disciplina basata su canoni ben precisi, ed è strettamente correlata alla psicologia (Longo M.A., 2013, pag. 9). E' necessario però specificare che essa è un test proiettivo mirato al tratto di personalità e non alla diagnosi, differentemente da come avviene nel colloquio clinico e con i test di tipo psicodiagnostici, e per tale motivo è importante la contestualizzazione, ossia esaminare tutti gli elementi tenendo presente lo stato d'animo del momento in cui la persona ha rilasciato la propria scrittura. Ciò non tanto perché lo stile del soggetto possa cambiare, ma perché nel tempo sicuramente esso potrà affinare alcuni aspetti del tratto caratteriale, in base alle varie esperienze di vita (Mastronardi V., Calderaro M., Leo S., 2021, pag. 211-212). Ovviamente in alcuni casi la disamina psicografologica va svolta con il cosiddetto approccio integrato, dove ogni professionista dà il suo contributo, sia nei casi in cui il reato o la condotta sia già avvenuta, sia nei casi in cui, grazie al riconoscimento dei cosiddetti "segnali di allarme" si sia in grado di intervenire prima di un comportamento finale e prevenire determinati accadimenti (Mastronardi V., Calderaro M., Leo S., 2021, pag. 220221). Pertanto la disamina della grafia può essere un valido supporto ad altri test compiuti durante la valutazione psicodiagnostica effettuata sul soggetto da psichiatri e psicologi psicodiagnosti. Alcuni esempi di test tra i più utilizzati in sede di valutazione sono: ${ }^{25}$
1. il MMPI-2 (Minnesota Multiphasic Personality Inventory), test utilizzato per diagnosticare alcuni problemi di personalità. Le risposte di tale test sono valutate in una scala dicotomica del tipo "vero-falso" che porta ad ottenere dei profili psicopatologici della persona che si somministra al test;

2. il test di Rorschach, utilizzato per indagare su diverse aree della personalità, da quella affettivo-relazionale, sessuale, all'approccio genitoriale, ecc. Tale test è in grado di dare indicazioni su disturbi d'ansia, fobie varie, disturbi del tono dell'umore, disturbi depressivi, psicosi anche grave, e consiste nel sottoporre al soggetto delle tavole, ognuna delle quali è caratterizzata da una macchia di inchiostro monocromatica o colorata;

3. il DFU (Disegno della Figura Umana), la sua funzionalità principale è quella di esprimere, attraverso il disegno, dinamiche interiori inconsce e inespresse. In questo test viene richiesto al soggetto di disegnare una figura che sia una proiezione dell'immagine di sé e permette di ottenere un quadro abbastanza ampio della sua personalità;

4. il Millon (Millon Clinical Multiaxial Inventory), anch'esso utilizzato per valutare dei disturbi di personalità e l'adattamento della persona. Questo test è anche strutturato per osservare come l'individuo funzioni a livello di interazione con l'ambiente e di quanto quest'ultimo possa influenzare il comportamento del

25 https://www.beatricecaponi.it (vedi Sitografia). 
soggetto, e le risposte, come nel MMPI-2, sono rilevate tramite una scala dicotomica "vero-falso".

Pertanto l'indagine psicografologica corrisponde ad un test proiettivo di personalità, poiché più il soggetto scrive, più emergeranno i "pattern grafologici" caratterizzanti la grafia, dal momento in cui viene meno il controllo della persona, la quale si lascia andare ai gesti che più la rappresentano. I tratti e i modelli di personalità più frequenti nell'ambito del comportamento antisociale, secondo Anthony Pinizzotto (1984), agente dell'FBI, sono: egocentrismo, impulsività, aggressività, dipendenza, frustrazione, narcisismo, paranoia, compulsività ossessiva (Mastronardi V., Calderaro M., Leo S., 2021, pag. 213-214). Dal punto di vista psicografologico, il tratto della dipendenza, il quale è il più rilevante dal punto di vista di questo lavoro di analisi sulla tossicodipendenza e sulla personalità del soggetto dipendente così come indicato precedentemente, a livello grafologico, possiede tra gli altri, le seguenti caratteristiche: invadenza del foglio con gesti ipertrofici nelle maiuscole, asole gonfie, soprattutto in riferimento alla lettera " $\mathrm{f}$ "; dimensioni grandi, da verticale a inclinata a destra fino a distendersi in alcune lettere; scrittura con poco spazio fra le parole, arrotondata, con tratti corti in finale di parola; fissazione alla fase orale (in riferimento alla psicoanalisi, primo stadio pulsionale chiamato "orale" proprio perché la zona che viene maggiormente stimolata è la bocca) (Mastronardi V., Calderaro M., Leo S., 2021, pag. 217). Data quindi l'importanza della grafologia come disciplina, è interessante come questa possa essere applicata anche nel caso in cui ci si trovi di fronte ad una persona tossicodipendente, dipendente, o presunta tale, poiché questo ulteriore strumento di analisi può facilitare la costruzione di un profilo comportamentale della persona in esame, al fine di individuare i tratti principali della personalità e le motivazioni più inconsce che spingono il soggetto alla dipendenza.

\section{Le nuove forme di dipendenza}

Le nuove forme di dipendenza trattate in questo capitolo rappresentano una novità, poiché non ci troviamo di fronte a dipendenze causate da una sostanza nociva, bensì a comportamenti anomali adottati dagli individui (Corbelli L., 2012, pag. 15-16). Esse vengono anche chiamate "dipendenze senza sostanza" e le principali sono:

- il gioco d'azzardo patologico, introdotto all'interno del DSM5 e definito come un "comportamento problematico persistente o ricorrente legato al gioco d'azzardo che porta a disagio o compromissione significativi..." (American Psychiatric Association, DSM-5, 2014, pag. 681);

lo shopping compulsivo, chiamato anche "oniomania" (mania del comprare). Esso rientra all'interno delle nuove dipendenze anche se la definizione non risulta semplice, dal momento in cui si colloca nel campo delle normali attività, socialmente accettata e condotta da tutti gli individui (Corbelli L., 2012, pag. 35);

la dipendenza da Internet, Internet Addiction Disorder (IAD), la quale si riferisce a tutte le condotte relative ad attività svolte in rete. Tale disturbo è stato inserito all'interno del DSM-5 nella sezione III, ossia quella riservata alle "condizioni che necessitano di ulteriori studi", proprio per la natura complessa di tale dipendenza. La dipendenza da Internet ovviamente si manifesta anche in culture diverse da quella europea, pertanto merita senz'altro una menzione la "Sindrome di Hikikomori", la quale è emersa in Giappone e letteralmente significa "isolarsi". Essa consiste nel ritiro della persona dal mondo reale per un periodo spropositato di tempo, e durante il quale l'unica forma comunicativa è rappresentata dal computer. Probabilmente la ragione che conduce a questo radicale isolamento è strettamente collegata alle caratteristiche della cultura giapponese, ovvero la forte competizione, le rigide gerarchie, le aspettative sociali. Tale sindrome è una manifestazione psicopatologica molto grave, poiché gli individui che ne sono affetti sono per la maggior parte adolescenti, i quali decidono volontariamente di recludersi nelle loro stanze, allontanandosi dai propri famigliari, dagli amici, per diversi mesi, talvolta anni. È necessario dunque monitorare il comportamento dei giovani, al fine di prevenire l'insorgenza di un disturbo psicopatologico di questo tipo (Corbelli L., 2012, pag. 100);

- la dipendenza affettiva, viene definita come uno specifico coinvolgimento in una relazione, la quale tende a sviluppare tolleranza e ripetitività. Le persone che sviluppano questo tipo di dipendenza sono coinvolte in rapporti in cui cercano di compiacere l'altro annullando la loro persona, al fine di soffocare un senso personale di inadeguatezza (Corbelli L., 2012, pag. 105-106);

- la dipendenza sessuale (sexual addiction) è una relazione patologica con il sesso, tramite la quale la persona ricerca un piacere che possa alleviare il suo stato d'animo negativo, lo stress. Il sesso quindi rappresenta un rifugio che permette alla persona di fuggire da una quotidianità basata su convinzioni disfunzionali (Caretti V., La Barbera D., 2005, pag. 168-169); la dipendenza da sport, chiamata anche "sindrome da overtraining", che consiste in un alterato equilibrio tra quantità e qualità di allenamento sportivo e tempi di recupero. La ricerca ossessiva di "raggiungere il miglior risultato" o del "dimagrire" può invalidare significativamente i sistemi di controllo endocrinologico; ${ }^{26}$

una forma di dipendenza che vale la pena citare in questo contesto è l'ortoressia, intesa come nuova condizione che si caratterizza per un comportamento alimentare che ha importanti conseguenze patologiche, ma che ancora non rientra all'interno del DSM-5. Il quadro clinico si caratterizza per la presenza di una ideazione prevalente sul controllo del regime alimentare, sulla scelta del cibo e sulle sue caratteristiche (dando l'assoluta priorità a cibi ritenuti "sani"), al fine di costruire una condotta nutrizionale che sia in grado di permettere al soggetto di raggiungere un "perfetto" stato di salute. ${ }^{27}$

Infine è stato interessante prendere in considerazione le differenti dinamiche delle dipendenze (da sostanze stupefacenti e non) e come queste siano cambiate durante il periodo di reclusione dovuto alla pandemia da Covid-19. Consultando la Relazione Annuale al Parlamento sul fenomeno delle tossicodipendenze in Italia relativa all'anno 2021(dati 2020), della Presidenza del Consiglio dei Ministri, all'interno del Dipartimento per le Politiche Antidroga, le droghe maggiormente sequestrate (sotto forma di polvere) durante l'anno 2020 sono state principalmente la cannabis, seguita dalle sostanze sintetiche e dalla cocaina, e per finire l'eroina e altri oppiacei, così come si può evincere dalla Fig. 1.

In merito alle NPS, nella Relazione Annuale al Parlamento sopracitata, è stato rilevato che nel primo semestre post-lockdown vi è stato un incremento significativo delle segnalazioni e delle intossicazioni da esse prodotte, a dimostrazione del fatto che la pandemia ha acutizzato il mercato delle Nuove Sostanze Psicoattive, poiché più facilmente reperibili sul web, da casa.

26 https://www.jpsychopathol.it (vedi Sitografia).
27 Ibidem. 


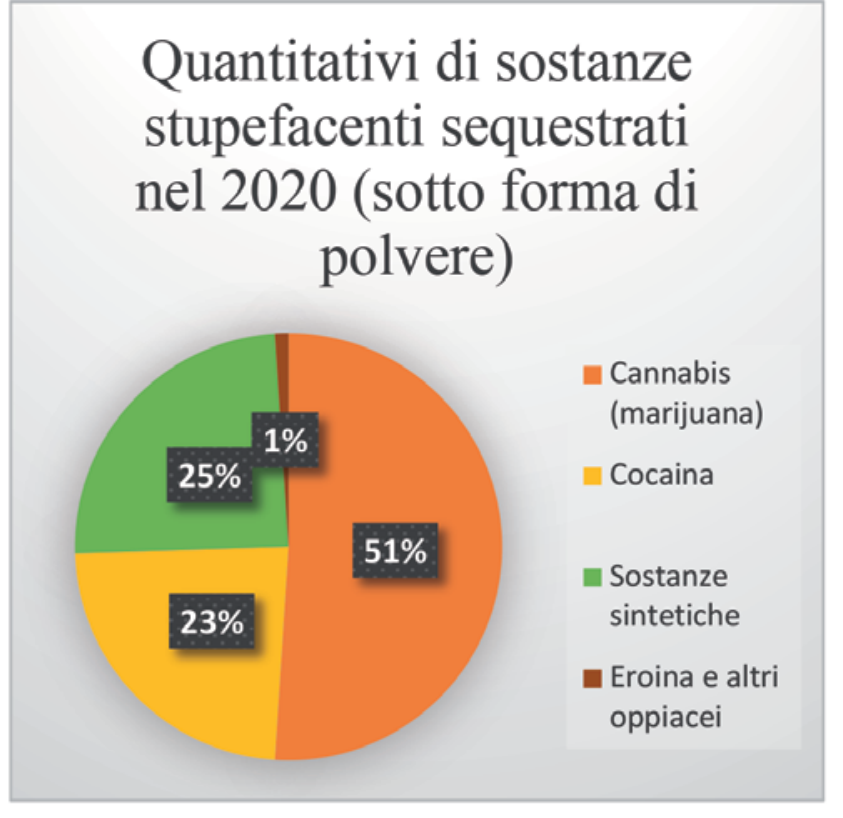

Fig. 1.

\section{Crescita della spesa del gioco online nel mese di marzo 2020}

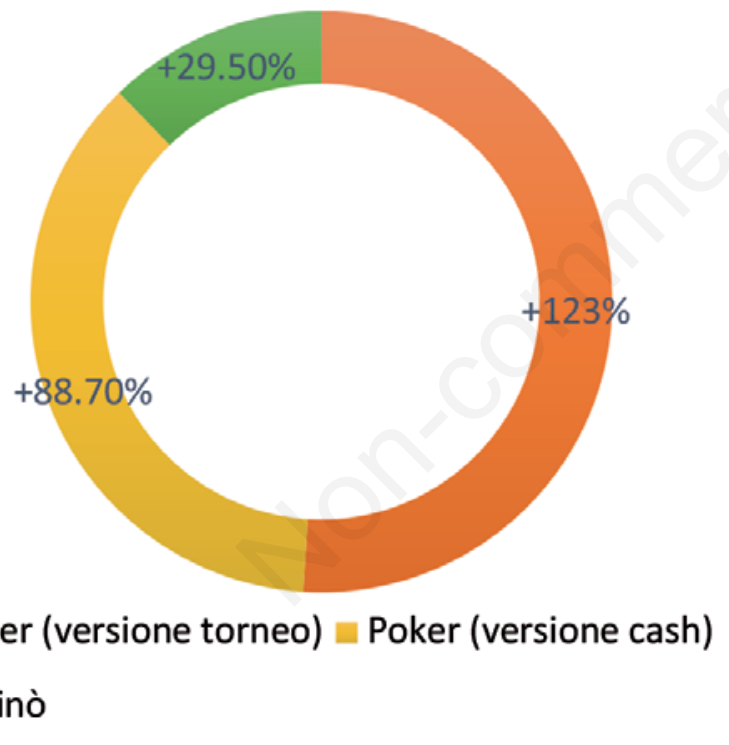

Fig. 2.

Inoltre il consumo di sostanze psicoattive da parte dei giovani è stato consistente: escludendo la cannabis, molti giovani hanno riferito di aver consumato almeno una sostanza illecita tra cocaina, stimolanti, eroina, inalanti.

Per ciò che concerne le dipendenze comportamentali, più precisamente il gioco online, l'Agenzia Giornalistica sul Mercato del Gioco (AGIMEG), ha preso in esame i dati in crescita relativi alla spesa degli italiani nel poker nella sua versione a torneo, nel poker in modalità cash (giocato con denaro reale o gettoni) e nel casinò online, mostrando come abbiano beneficiato tali attività durante il periodo di restrizione nel 2020, così come si evince dalla Fig. 2.

\section{Persone affette da disturbi \\ alimentari}

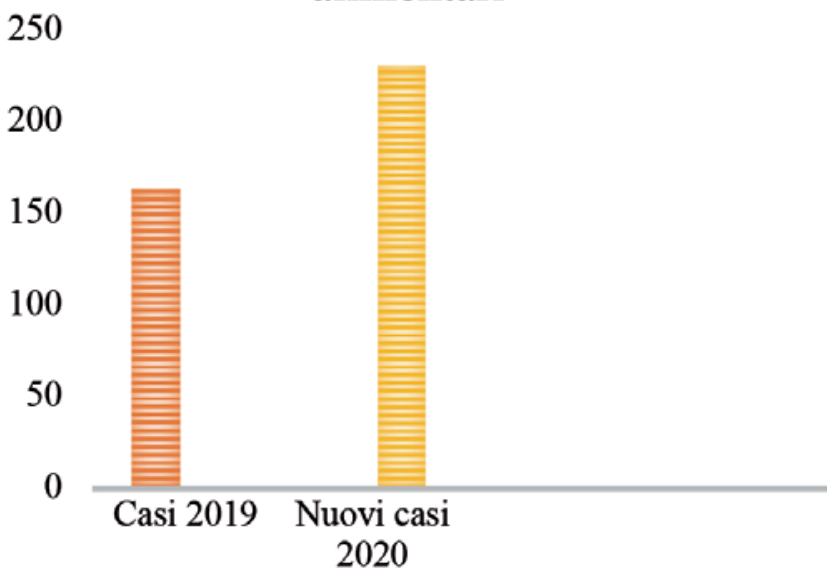

Fig. 3.

L'istituto Superiore di Sanità ha preso in considerazione anche i disturbi dell'alimentazione come anoressia, bulimia nervosa e alimentazione incontrollata (il binge eating) in relazione ai rischi di insorgenza causati anche dalla pandemia da Covid-19. ${ }^{28}$ Tali patologie rientrano all'interno dei Disturbi del Comportamento Alimentare (DCA) e sono un fenomeno ancora del tutto sottovalutato in Italia, ma che ha subito una crescita drastica proprio durante il periodo di isolamento. Dai dati resi noti dal Centro Nazionale per il Controllo e la Prevenzione delle Malattie, nei primi sei mesi del 2020 , l'Italia ha visto crescere il fenomeno dei disturbi alimentari del 30\%, così come mostrato dalla Fig. 3 .

Un ulteriore elemento da considerare, emerso con più facilità a causa delle restrizioni dovute alla pandemia, è il fenomeno del binge watching ("abbuffata di visione"). Tale termine si è sempre più diffuso in Europa con l'avvento di piattaforme streaming come Netflix e Amazon Prime Video, le quali offrono un modo differente di guardare serie tv e film. In inglese infatti il termine binge watching indica proprio la visione consecutiva di puntate di una serie tv, rimanendo così per lungo tempo davanti alla televisione. Nella maggior parte delle piattaforme le puntate successive partono in automatico e ciò sicuramente non aiuta i soggetti a "staccarsi" dai vari stimoli visivi. Un'altra pratica molto diffusa è il binge racing ("gara d'abbuffata"), la quale consiste nel guardare tutti gli episodi di una serie appena uscita entro le 24 ore successive al lancio ed è praticata soprattutto da adolescenti e giovani adulti. Le pratiche sopradescritte possono portare a criticità significative come vissuti di forte ansia, depressione o sensi di vuoto in concomitanza con la fine delle serie tv. E' perciò importante ricordare che esse sono molto pericolose per la salute e il corpo degli individui e non devono essere sottovalutate. Le persone non si rendono conto effettivamente delle ore che passano a guardare film e serie tv e questo comporta una sovrastimolazione cerebrale che rende difficoltoso il sonno. Pertanto anche questi fenomeni devono essere efficacemente analizzati, poiché potrebbero condurre a delle dipendenze comportamentali. ${ }^{29}$

28 https://www.epicentro.iss.it (vedi Sitografia).

29 https://psicologiapsicosomatica.com (vedi Sitografia). 


\section{Conclusioni}

La tecnica del profiling in criminologia è senz'altro un valido aiuto per coloro che vogliono comprendere la natura più profonda di un comportamento e rintracciare il colpevole di un delitto. Tuttavia in questo elaborato tale tecnica viene considerata con un differente approccio, partendo da una specifica domanda: essa può essere applicata anche nel caso in cui ci si trovi di fronte ad una persona dipendente o presunta tale? Ovviamente nel caso in cui si abbia a che fare con un soggetto tossicodipendente non si ha né una scena del delitto, né una vittima, né un colpevole; o meglio vittima e colpevole possono forse essere rintracciati nella medesima persona. Perciò anche se il profiling in questo contesto non può essere applicato secondo le procedure ordinarie, sicuramente vi può essere una modalità che aiuti chi si occupa di individui tossicodipendenti. Andare ad individuare i segnali della dipendenza e le cause di quest'ultima può senz'altro aiutare ad inquadrare meglio il "perché" di un determinato comportamento, in questo caso considerando sia le tossicodipendenze (nello specifico quelle derivanti dalle Nuove Sostanze Psicoattive), sia le nuove forme di dipendenza, chiamate anche dipendenze comportamentali o dipendenze senza sostanza, le quali rappresentano una novità, poiché non si riferiscono ad una sostanza psicoattiva tossica per l'organismo dell'individuo. Un ulteriore strumento di supporto è la già citata disamina psicografologica, la quale permette di analizzare il comportamento in un'ottica preventiva, nel caso in cui il soggetto non abbia ancora esplicitato il suo comportamento dipendente. Ad ogni modo tentare di approcciare la tossicodipendenza con la tecnica del profiling potrebbe essere utile a chi si occupa del fenomeno, specialmente perché la dipendenza è uno dei tratti di personalità che possono causare un comportamento antisociale e per tale motivo, se efficacemente analizzata, può portare ad una comprensione del fenomeno più ampia e magari, chissà, riuscire ad evidenziare quelle condotte tipiche e riscontrabili solo nelle persone che presentano determinate specificità.

\section{Bibliografia}

American Psychiatric Association, (2014), Manuale Diagnostico e Statistico dei disturbi mentali DSM-5, Cortina Editore, Milano.

Calderaro M., Leo S., Mastronardi V., (2021), Staging e scena del crimine. Manuale di analisi comportamentale, Carocci Editore, Roma.

Caretti V., La Barbera D., (2005), Le dipendenze patologiche. Clinica e psicopatologia, Cortina Editore, Milano.

Corbelli L., (2012), Nuove dipendenze. Guida ad un fenomeno complesso, Aras Edizioni, Fano.

Di Giannantonio M., Girardi P., (2016), Psicopatologia delle dipendenze, Pacini Editore, Pisa.

Douglas J., (2017), Mindhunter, Longanesi, Edizione 2017, Milano.

Longo M., A., (2013), Manuale pratico di grafologia e caratterologia, Hermes Edizioni, Roma.

Marazziti D., Presta S., Picchetti M., Dell'Osso L., (2015), Behavioral addiction: clinical and therapeutic aspects, J Psycopathol 21:72-84. https://www.jpsychopathol.it/issues /2015/vol21-1/10Marazziti.pdf

OHagan A., Smith C., (2017), A new beginning: an overview of New Psychoactive Substances, in: Forensic Res Criminol Int J 5:00159.

Palermo G.B, Mastronardi V.M, (2005), Il profilo criminologico.
Dalla scena del crimine ai profili socio-psicologici, Giuffrè Editore, Milano.

Rinaldi R., Bersani G., Marinelli E., Zaami S., (2020), The rise of new psychoactive substances and psychiatric implications: a wide - ranging, multifaceted challenge that needs far- reaching common legislative strategies, Hum Psychopharmacol 35:e2727.

\section{Sitografia}

AGIMEG, (2020), Chiusura agenzie scommesse, esercizi con slot, sale gioco e bingo e giochi in tabaccheria ma l'online tra alti e bassi: bene poker e casinò, crollano le scommesse sportive, in: https://www.agimeg.it/datitalia/agenzie-scommesse-chiusurapoker-cash-torneo-casino-coronavirus

Bignamini E., (2021), Termini utili e definizioni per capire meglio la dipendenza, in: https://magazine.istitutoeuropeodipendenze.it/ termini-utili-e-definizioni-per-capire-meglio-la-dipendenza/.

Canoniero S., Meremmani I., Pacini M., (2002), Psico(pato)logia dell'"addiction". Un'ipotesi interpretativa, Pisa, Annali Istituto Superiore di Sanità: https://www.iss.it/documents/20126/955767/ 383241.1108646898.pdf/369cb5ba-8dc0-177e-3835-8434e5129c af? $\mathrm{t}=1575579464542$

Caponi B., Valutazione psicodiagnostica, in: https://www. beatricecaponi.it/attivita/valutazione-psicodiagnostica-torrevec chia-roma.html

Castello E., Afflitto H., Maggio D., (2021), Binge watching: quando l'abbuffata da serie tv può diventare un pericolo? in: https://psicologiapsicosomatica.com/2021/06/23/binge-watchingquando-labbuffata-da-serie-tv-puo-diventare-un-pericolo/

Dipartimento per le Politiche Antidroga, Presidenza del Consiglio dei Ministri (2021), Relazione annuale al Parlamento sul fenomeno delle tossicodipendenze in Italia anno 2021(dati 2020), in: https:// www.politicheantidroga.gov.it/media/3076/rap2021pdf.pdf

European Monitoring Centre for Drugs and Drug Addiction (EMCDDA), Focus sulle droghe. Briefing dell'Osservatorio europeo delle droghe e delle tossicodipendenze. Droghe e criminalità: un rapporto complesso, in: https://www.emcdda.e uropa.eu/system/files/publications/470/Dif16IT_84993.pdf

International Drug Policy Consortium (IDPC), L'INCB (International Narcotics Control Board), in: https://idpc.net/it/advocacy/ advocacy-a-livello-globale/sistema-globale-di-controllo-delle-dro ghe/incb

Istituto Superiore di Sanità, (2020), Disturbi dell'alimentazione e Covid-19, in: https://www.epicentro.iss.it/coronavirus/sars-cov2-dipendenze-disturbi-alimentazione

Ministero dell'Interno, (2014), Le nuove sostanze psicoattive (NSP), in: https://www.interno.gov.it/sites/default/files/allegati/schede droghe.pdf

Ministero dell'Interno, Dipartimento della Pubblica Sicurezza, Direzione Centrale per i Servizi Antidroga, (2021), Relazione annuale 2021 (dati 2020), in: https://antidroga.interno.gov.it/wpcontent/uploads/2021/07/Relazione-Annuale-2021.pdf

Ministero della Salute, Direzione Generale della Prevenzione Sanitaria (2020), Piano Nazionale della Prevenzione 2020-2025, in: https://www.salute.gov.it/imgs/C_17_pubblicazioni_2955 allegato.pdf

Ministero della Salute (2021), Tabelle delle sostanze stupefacenti e psicotrope, in: https://www.salute.gov.it/portale/temi/p2_6.jsp? $\mathrm{id}=3729 \&$ are $=$ sostanzeStupefacenti\&menu $=$ vuoto

Ministero della Salute (2021), Decreto 6 Agosto 2021, in: https://www1.ordinemediciroma.it/images/URP/allegato_176.pdf 
Ministero della Salute (2021), Decreto 2 Dicembre 2021, in: https://www1.ordinemediciroma.it/images/URP/ALL_73.pdf

Progetto NPS 2018 - Multicentrica di Ricerca DPA- Presidenza del Consiglio dei Ministri, NPS Alert. New Psycoactive Substances Advanced Research, in: http://allerta.dronetplus.eu/nsp.html

Segretariato generale del Consiglio dell'Unione Europea, (2020), Strategia dell'Unione Europea in materia di droga per il periodo 2021-2025, in: https://www.politicheantidroga.gov.it/media/3008/ strategia-ue-2021-2025-ita.pdf

Sky TG24 (2021), Oggi è la Giornata nazionale dei disturbi del comportamento alimentare, in: https://tg24.sky.it/salute-ebenessere/2021/03/15/disturbi-comportamento-alimentare-giorna ta-nazionale-2021

Università Cattolica del Sacro Cuore, (2013), Cos'è il poliabuso, Cattolica News, in: https://www.cattolicanews.it/alte_scuole/ poliabuso.pdf

Correspondence: Monica Calderaro.

E-mail: calderaromonica6@gmail.com

Key words: drug addiction; profiling; new psychoactive substances. Parole chiave: tossicodipendenza; profiling; nuove sostanze psicoattive. Palabras clave: toxicomanía; profiling; nuevas sustancias psicoactivas.

Received for publication: 1 February 2021.

Accepted for publication: 13 March 2021.

This article is distributed under the terms of the Creative Commons Attribution Noncommercial License (by-nc 4.0) which permits any noncommercial use, distribution, and reproduction in any medium, provided the original author(s) and source are credited.

${ }^{\circ}$ Copyright: the Author(s), 2021

Licensee PAGEPress, Italy

Rivista di Psicopatologia Forense, Medicina Legale, Criminologia $2021 ; 26: 614$

doi:10.4081/psyco.2021.614 